\title{
Utilização de plantas medicinais como remédio caseiro na Atenção Primária em Blumenau, Santa Catarina, Brasil
}

\author{
Use of medicinal plants as home remedies in Primary Health Care \\ in Blumenau - State of Santa Catarina, Brazil
}

Ana Lúcia Bertarello Zeni ${ }^{1}$
Amanda Varnier Parisotto $^{2}$
Gerson Mattos $^{1}$
Ernani Tiaraju de Santa Helena ${ }^{1}$

${ }^{1}$ Programa de PósGraduação em Saúde Coletiva, Universidade Regional de Blumenau (FURB). R. Antônio da Veiga 140, Itoupava Seca. 89030-903 Blumenau SC Brasil. anazeni@furb.br ${ }^{2}$ Curso de Medicina, Departamento de Medicina, FURB. Blumenau SC Brasil.

\begin{abstract}
An increase in the use of alternative therapeutic practices has been observed in the past decade, especially in medicinal plants, herbal and home remedies, which has been supported by policies within the scope of the Unified Health System (SUS). This study investigated the use of home remedies by users of Primary Health Care in Blumenau, State of Santa Catarina. It is a cross-sectional, observational and epidemiological study, the data for which were obtained via a questionnaire applied to 701 individuals. An unconditional logistic regression model was used to estimate the association between the use of home remedies and socio-demographic and medical care variables. It was observed that $21.9 \%$ of the sample use home remedies and medicinal plants grown in the back yard are the remedies of choice. Lemon balm, chamomile, peppermint and lime were the remedies most frequently mentioned. The use of home remedies was associated with the female gender, older age and the Family Health Strategy care model. The results supported that medicinal plants are used by the population as a therapeutic alternative option. However, it is necessary that primary care services ensure both access to natural products and supply qualified professionals to give instructions regarding the correct usage of home remedies.
\end{abstract}

Key words Primary Health Care, Medicinal plants, Complementary therapies
Resumo Na última década foi observado um aumento no uso de práticas terapêuticas alternativas apoiadas por políticas no âmbito do Sistema Único de Saúde (SUS), em particular o uso de plantas medicinais e de fitoterápicos. Este estudo investigou o uso de remédios caseiros pelos usuários da Atenção Primária da Saúde do município de Blumenau, em Santa Catarina. Estudo epidemiológico observacional seccional, cujos dados foram obtidos através de questionário aplicado a 701 indivíduos. Utilizou-se um modelo de regressão logística não condicional para estimar a associação entre uso de remédios caseiros e variáveis sociodemográficas e médicoassistenciais. Observou-se que $21,9 \%$ dos entrevistados utilizaram remédios caseiros, sendo as plantas medicinais obtidas no quintal das casas a principal escolha. Como as mais citadas destacaram-se erva-cidreira, camomila, hortelã e limão. O uso de remédios caseiros se mostrou associado ao sexo feminino, à idade mais avançada e à modalidade de serviço, Estratégia Saúde da Família. Os resultados mostraram que as plantas medicinais são utilizadas como alternativa terapêutica. Entretanto, é necessário que os serviços de atenção primária garantam o acesso aos produtos naturais, bem como profissionais qualificados capazes de fornecer orientações sobre sua utilização.

Palavras-chave Atenção Primária à Saúde, Plantas medicinais, Terapias complementares 


\section{Introdução}

Práticas Integrativas e Complementares (PICs) compreendem um conjunto de sistemas, práticas e produtos de uso clínico, não considerado como prática médica convencional. Após a publicação da Política Nacional de Práticas Integrativas e Complementares (PNPIC), a homeopatia, as plantas medicinais e fitoterápicos, a medicina tradicional chinesa/acupuntura, a medicina antroposófica e o termalismo social-crenoterapia foram institucionalizados no Sistema Único de Saúde (SUS) ${ }^{1}$. Neste sentido, a Organização Mundial da Saúde (OMS) define Medicina Tradicional (MT) e Medicina Alternativa e Complementar $(\mathrm{MAC})^{2}$ como práticas que incluem terapias com medicação, as quais usam ervas, partes de animais ou minerais, e terapias sem medicação, por exemplo, a acupuntura, terapias manuais e espirituais.

Vários estudos demonstram o uso das práticas compreendidas na MAC em diversas partes do mundo, sendo que na França este percentual chegou a 75\%, no Canadá 70\% e nos EUA $42 \%{ }^{2}$. A demanda cada vez maior por tratamentos alternativos está relacionada à percepção das limitações da medicina convencional, aumentos das doenças iatrogênicas e crônicas, enfraquecimento da relação médico-paciente e busca por atenção integral à saúde ${ }^{3}$, além da questão financeira e de inclusão social relacionadas a essas terapias ${ }^{4}$.

Diante da grande difusão dessas práticas surgiu a necessidade de integrá-las às políticas públicas de saúde. A OMS orienta a inclusão deste tipo de terapia no sistema público, desde 1979, por ocasião da Conferência Internacional de Alma $\mathrm{Ata}^{2}$. No Brasil, foi elaborado a PNPIC para o SUS em 2006. Esse projeto consiste de uma política pública que visa ampliar o atendimento na Atenção Básica à Saúde, através da utilização das práticas integrativas e complementares à medicina convencional ${ }^{5}$.

A inclusão de tais práticas na atenção primária deve seguir os princípios norteadores do SUS aceitando o compromisso de proporcionar assistência universal, integral, equânime, contínua e resolutiva à população, de acordo com as necessidades, por meio da identificação dos fatores de risco aos quais está exposta e neles interferir de forma pertinente ${ }^{4}$. Além disso, existe a necessidade de abordar a realidade multifacetada em que os indivíduos são alvos das ações em saúde. O conhecimento científico deve agregar os elementos dessa realidade, mesmo os mais simples e considerados “irrelevantes”, pois as diferenças culturais e sociais de cada população se refletem no processo de saúde-doença e alteram os resultados das ações tomadas pelos profissionais da área da saúde. Minayo ${ }^{6}$ afirma que a vivência da saúde e da doença traz mudanças para o corpo e o espírito, por isso os profissionais de saúde precisam considerar os valores e as crenças das pessoas, ampliando seus conceitos e tornando mais inclusiva suas formas de abordar os problemas do cotidiano em que atuam. Desta forma, os serviços que oferecem práticas não usuais podem contribuir para que a clientela mais satisfeita utilize menos retornos, exames diagnósticos e encaminhamento para os níveis secundário e terciário 3 .

O Programa Nacional de Plantas Medicinais e Fitoterápicos visa "Promover e reconhecer as práticas populares e tradicionais de uso de plantas medicinais, fitoterápicos e remédios casei-

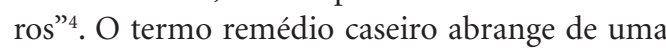
forma bastante ampla a utilização de ervas, partes de animais ou minerais, para fins terapêuticos preparados em ambiente caseiro. Entretanto, Oliveira et al. ${ }^{7}$ mostram a prevalência do uso de planta medicinal como forma de PIC, corroborando dados levantados pela OMS, os quais apontam que $80 \%$ da população mundial faz uso de algum tipo de planta em busca de alívio para sua sintomatologia ${ }^{8}$. A alta incidência de uso de plantas medicinais possivelmente deve-se ao fácil acesso, baixo custo e por serem consideradas inofensivas por grande parte da população ${ }^{9}$. Além disso, medicamentos industrializados são caros, enquanto plantas medicinais muitas vezes são cultivadas nos quintais ${ }^{10}$.

Porém, geralmente o conhecimento de plantas com fins terapêuticos é praticado sem acompanhamento médico, representando um perigo potencial para a população, pois existe a possibilidade de interação entre esses produtos "naturais” e os medicamentos, além da interferência dos mesmos em resultados de exames laboratoriais $^{11,12}$. Neste sentido, a orientação vinda do profissional da saúde é fundamental para que o paciente possa ser alertado sobre os riscos da toxicidade, interações medicamentosas e melhores formas de utilização das terapias alternativas.

O presente estudo teve como objetivo avaliar o uso de remédios caseiros na Atenção Primária no município de Blumenau e as variáveis que influenciaram na escolha deste tipo de terapia pelos usuários. 


\section{Métodos}

\section{Desenho do estudo}

Trata-se de um estudo epidemiológico observacional, tipo seccional, que utilizou dados obtidos pelo projeto "Melhoria da qualidade da assistência farmacêutica na Atenção Básica do SUS, Blumenau, SC”.

\section{População de estudo e amostra}

A população de estudo foi composta por pessoas atendidas nas farmácias das unidades de atenção primária de Blumenau. A Atenção Primária à Saúde (APS) é considerada a porta de entrada do SUS. Dentre as modalidades de APS em Blumenau, destacam-se as Unidades Básicas com equipes de Estratégia Saúde da Família (ESF) e os Ambulatórios Gerais (AG). As equipes de ESF são compostas por médico, enfermeira, técnicos de enfermagem e agentes comunitários de saúde. Os AG contam com clínico-geral, pediatra, gineco-obstetra, profissionais de enfermagem, psicólogo, assistente social e farmacêutico. Ambas têm equipes de saúde bucal. Blumenau contava com $46 \%$ da população coberta por equipes de ESF em 2010.

A amostra foi obtida em dois estágios - unidades e usuários - proporcionalmente pelo número total de usuários assistidos pela respectiva unidade. O tamanho da amostra deveria ser capaz de estimar uma proporção de $50 \%$ de pessoas que utilizam remédios caseiros, com precisão de $5 \%$, intervalo de confiança de $95 \%$ e efeito desenho igual a 2, totalizando 768 pessoas. Os usuários que compareceram às farmácias das unidades de saúde para obter medicamentos foram incluídos na amostra após assinarem o Termo de Consentimento Livre e Esclarecido (TCLE). No caso de menores de 18 anos, os TCLE eram assinados pelos pais ou responsáveis. Foram excluídos aqueles que apresentassem alguma limitação física ou cognitiva que comprometesse as respostas do questionário.

O trabalho de campo foi realizado em 3 AG e 11 ESF. As Unidades de Saúde com equipes de ESF e os AG localizam-se em diversas áreas da cidade de Blumenau, sendo que cada um deles pertence a uma região diferente e todos os locais são considerados áreas urbanas.

As informações foram obtidas por entrevistadores previamente treinados e registradas em questionários estruturados e predominantemente fechados, os quais foram aplicados no período de setembro de 2009 a abril de 2010 . Informações referentes a menores de 18 anos foram obtidas a partir dos responsáveis legais.

\section{Análise dos dados}

Foram estudadas as variáveis: sexo, idade (em anos completos e por faixas etárias), estado civil, escolaridade (em anos de estudo completos), cor autodeclarada, classe econômica de consumo, doenças de base, tempo de tratamento, quantidade de medicamentos usados nos últimos 15 dias, se faz uso de medicamentos de uso contínuo e se necessitou ou não internação hospitalar nos últimos 12 meses. As doenças foram classificadas e agrupadas de acordo com Classificação Internacional de Doenças e Problemas Relacionados à Saúde - décima revisão Doenças (CID-10) da $\mathrm{OMS}^{13}$. A classe econômica de consumo foi definida com base nos critérios da Associação Brasileira de Empresas de Pesquisa $(\mathrm{ABEP})^{14}$.

A variável de desfecho foi "uso de remédio caseiro" entendido como todo e qualquer tipo de cuidado utilizado para curar ou aliviar doenças, sintomas, desconforto e mal-estar. Além disso, questionou-se o tipo de remédio, o local de obtenção, a forma de uso e o motivo pelo qual foi utilizado. Esta pesquisa não objetivou um estudo etnobotânico, com coleta de plantas visando à identificação botânica completa e a comprovação do uso científico de plantas utilizadas pela população.

Os dados foram digitados com dupla entrada no software EPI-DATA (domínio público) e feito controle de qualidade de possíveis erros. Inicialmente, as variáveis foram analisadas de maneira descritiva, através de cálculo de frequências, com os respectivos intervalos de 95\% de confiança e medidas de tendência central e variabilidade.

Foi calculada a prevalência de uso de remédio caseiro com intervalo de 95\% de confiança para uma distribuição binomial.

Foi calculado o "Odds Ratio" (OR), com intervalo de $95 \%$ de confiança, como medida de associação entre a variável utilização de remédio caseiro e as variáveis independentes sociodemográficas e médico-assistenciais. Na análise multivariada, calcularam-se os valores de OR por regressão logística não condicional. Utilizou-se a técnica de retirada paulatina das variáveis com base nos níveis de significância (stepwise backward), permanecendo aquelas que mantiveram um valor de $\mathrm{p}<0,05$, estimado pela Razão de 
Máxima Verossimilhança. Foi testado o ajuste do modelo final pelo teste de Hosmer-Lemeshow.

\section{Aspectos éticos}

Este estudo foi aprovado pelo Comitê de Ética em Pesquisas de Seres Humanos da Universidade Regional de Blumenau (FURB).

\section{Resultados}

Foram entrevistados 701 indivíduos, ocorrendo 9 perdas por informações incompletas e 58 por recusas, destes foram 548 mulheres $(78,1 \%)$ com média de idade de 43,3 anos $( \pm 19,2)$ e mediana de 43,8 anos, sem diferenças entre os sexos. A maioria $(569,81,6 \%)$ dos entrevistados declarou-se branca, casada $(394,56,4 \%)$ e $395(56,4 \%)$ pertencentes à classe $\mathrm{C}$. A média de anos de estudos foi de 6,9 anos $( \pm 3,5)$ e a mediana de 7 anos (Tabela 1).

A maioria das pessoas foi assistida em AG $(425,60,6 \%)$. Entre as afecções tratadas mais comuns nas Unidades de Saúde, destacaram-se as "doenças do aparelho circulatório" (161, 23,3\%) e as "doenças endócrinas, nutricionais e metabólicas" (13,1\%). A mediana de tempo de duração da doença de base foi de 12 meses. O número médio de medicamentos consumidos nos últimos 15 dias foi de 2,6 (mediana $=2$ ), enquanto 130 pessoas $(18,7 \%)$ necessitaram de internação hospitalar no último ano (Tabela 2). Dentre as 385 pessoas que utilizavam medicamentos continuamente, 57 pararam de usar por conta própria e, destas, 3 substituíram por remédios caseiros.

A prática de uso de remédio caseiro foi relatada por $151(21,8 \%)$ dos entrevistados, sendo que as plantas medicinais (96\%) foram a principal terapia escolhida, as demais respostas (4\%) mencionaram outras formas como: água com sal ou açúcar, gordura de capivara, leite e mel. Neste estudo, foi citado pelos entrevistados um total de 55 plantas, sendo que as 10 mais referidas estão apresentadas na Tabela 3.

Em relação ao local de obtenção das plantas, a maioria dos entrevistados coleta no próprio quintal $(76,51,0 \%)$, com amigos, familiares ou vizinhos $(19,12,8 \%)$, farmácia $(14,9,4 \%)$ ou mercado/loja de produtos naturais $(36,24,2 \%)$. Somente $4(2,7 \%)$ dos entrevistados relataram obter plantas na Unidade de Saúde. As formas de preparo das plantas medicinais informadas foram, chá $(87,4 \%)$, suco $(5,3 \%)$, fruta $(2,0 \%)$, xarope $(1,3 \%)$, pomada $(1,3 \%)$, compressas
Tabela 1. Caracterização sociodemográfica dos usuários atendidos pela Atenção Primária em Blumenau (SC).

\begin{tabular}{|c|c|c|}
\hline Variável & $\mathbf{N}$ & $\%($ IC95\%) \\
\hline \multicolumn{3}{|l|}{ Sexo $(n=701)$} \\
\hline Masculino & 153 & $21,8(18,8-25,1)$ \\
\hline Feminino & 548 & $78,1(74,9-81,2)$ \\
\hline \multicolumn{3}{|c|}{ Faixa etária $(\mathrm{n}=701)$} \\
\hline Menor que 20 anos & 87 & $12,4(10,1-15,1)$ \\
\hline 20 a 64 anos & 516 & $73,6(70,2-76,8)$ \\
\hline 65 anos e mais & 98 & $14,0(11,5-16,8)$ \\
\hline \multicolumn{3}{|c|}{ Cor autodeclarada $(\mathrm{n}=697)$} \\
\hline Branco & 569 & $81,6(78,6-84,4)$ \\
\hline Outros & 128 & $18,4(15,6-21,4)$ \\
\hline \multicolumn{3}{|c|}{ Estado civil $(\mathrm{n}=699)$} \\
\hline Solteiro & 182 & $26,0(22,8-29,5)$ \\
\hline Casado & 394 & $56,4(52,6-60,1)$ \\
\hline Separado & 50 & $7,2(5,4-9,3)$ \\
\hline Viúvo & 73 & $10,4(8,3-13,0)$ \\
\hline \multicolumn{3}{|c|}{ Escolaridade $(\mathrm{n}=687)$} \\
\hline Analfabetos & 25 & $3,6(2,4-5,3)$ \\
\hline 1 a 4 anos & 215 & $31,3(27,8-34,9)$ \\
\hline 5 a 8 anos & 207 & $30,1(26,7-33,7)$ \\
\hline 9 a 11 anos & 186 & $27,1(23,8-30,6)$ \\
\hline 12 e mais & 41 & $6,0(4,3-8,0)$ \\
\hline Não se aplica & 13 & $1,9(1,0-3,2)$ \\
\hline \multicolumn{3}{|c|}{ Classe de consumo $(\mathrm{n}=701)$} \\
\hline A & 8 & $1,1(0,5-2,2)$ \\
\hline B & 223 & $31,8(28,4-35,4)$ \\
\hline $\mathrm{C}$ & 395 & $56,4(52,6-60,1)$ \\
\hline $\mathrm{D}$ & 74 & $10,6(8,4-13,1)$ \\
\hline E & 1 & $0,1(0,0-0,7)$ \\
\hline
\end{tabular}

IC: intervalo de confiança

$(0,7 \%)$, gargarejo $(0,7 \%)$, garrafadas $(0,7 \%)$ e banhos de assento $(0,7 \%)$. A prática do preparo de garrafadas foi relatada apenas por um entrevistado, que mistura várias plantas buscando um efeito fortificante.

A Tabela 4 mostra a análise univariada da associação entre diversas variáveis das pessoas e médico-assistenciais e o uso de remédios caseiros. Observou-se associação de forma significativa para as variáveis: idade, estado civil, escolaridade, modalidade de serviço, tempo de doença de base e número de medicamentos consumidos.

O modelo de regressão logística ajustado encontra-se apresentado na Tabela 5. 
Tabela 2. Características das variáveis médico-assistenciais dos pacientes atendidos pela Atenção Primária em Blumenau (SC).

\begin{tabular}{|c|c|c|}
\hline Variável & $\mathbf{N}$ & $\%(\mathrm{IC} 95 \%)$ \\
\hline \multicolumn{3}{|l|}{ Modalidade APS $(\mathrm{n}=701)$} \\
\hline AG & 425 & $60,6(56,9-64,3)$ \\
\hline ESF & 276 & $39,4(35,7-43,1)$ \\
\hline \multicolumn{3}{|l|}{ Doencas de base - CID agrupada $(n=693)$} \\
\hline Doenças endócrinas, nutricionais e metabólicas & 91 & $13,1(10,7-15,8)$ \\
\hline Transtornos mentais e comportamentais & 50 & $7,2(5,4-9,4)$ \\
\hline Doenças do aparelho circulatório & 161 & $23,2(20,1-26,6)$ \\
\hline Doenças do aparelho respiratório & 52 & $7,5(5,6-9,7)$ \\
\hline Doenças do aparelho digestivo & 44 & $6,4(4,6-8,4)$ \\
\hline Doenças do sistema osteomuscular & 48 & $6,9(5,1-9,1)$ \\
\hline Sintomas, sinais mal definidos & 52 & $7,5(5,7-9,7)$ \\
\hline Demais causas & 195 & $28,1(24,8-31,6)$ \\
\hline \multicolumn{3}{|l|}{ Tempo da doença de base $(n=684)$} \\
\hline Até um ano & 360 & $52,6(48,8-56,4)$ \\
\hline Um ano e mais & 324 & $47,4(43,6-51,2)$ \\
\hline \multicolumn{3}{|l|}{ Número de medicamentos $(n=692)$} \\
\hline 0 a 2 & 395 & $57,1(53,3-60,8)$ \\
\hline 3 a 4 & 145 & $20,9(18,0-24,2)$ \\
\hline 5 e mais & 152 & $22,0(18,9-25,2)$ \\
\hline \multicolumn{3}{|l|}{ Medicamentos de uso contínuo $(\mathrm{n}=701)$} \\
\hline Sim & 385 & $54,9(51,2-58,7)$ \\
\hline Não & 316 & $45,1(41,4-48,8)$ \\
\hline \multicolumn{3}{|l|}{ Hospitalização nos últimos 12 meses $(\mathrm{n}=700)$} \\
\hline Sim & 130 & $18,6(15,8-21,7)$ \\
\hline Não & 570 & $81,4(78,3-84,2)$ \\
\hline
\end{tabular}

IC: intervalo de confiança; APS: atenção primária à saúde; AG: ambulatório geral; ESF: estratégia saúde da família; CID: classificação internacional de doenças e problemas relacionados à saúde.

Tabela 3. Relação das 10 plantas mais citadas (frequência relativa) e indicações de uso medicinal relatadas pelos usuários da Atenção Primária em Blumenau (SC).

\begin{tabular}{lrrl}
\hline \multicolumn{1}{c}{ Nome popular } & \multicolumn{1}{c}{ N } & \multicolumn{1}{c}{ \% } & \multicolumn{1}{c}{ Indicações populares } \\
\hline Erva-cidreira & 26 & 17,3 & "calmante, pra dormir e relaxar" \\
Camomila & 19 & 12,6 & "pra cólicas, dormir, calmante, dor de cabeça e baixar a pressão" \\
Hortelã & 14 & 9,3 & "calmante, gripe, dor de barriga" \\
Limão & 11 & 7,3 & "baixar pressão, gripe, pra não dar convulsão, dor" \\
Boldo & 10 & 6,6 & "dor de estômago, calmante, azia, má digestão" \\
Cana-de-cheiro & 9 & 6,0 & "pra baixar pressão, calmante" \\
Malva & 9 & 6,0 & "infecção na bexiga, gripe, dor, inflamação" \\
Erva-doce & 7 & 4,6 & "calmante, pra dormir" \\
Maracujá & 5 & 3,3 & "calmante, para não dar convulsão" \\
Laranja & 4 & 2,6 & "gripe" \\
Outros & 27 & 24,4 & ------------ \\
Total & 151 & 100 & \\
\hline
\end{tabular}

\section{Discussão}

O Programa Nacional de Plantas e Fitoterápicos visa a promoção e o reconhecimento das práticas populares e tradicionais como terapia, entretanto, não existe conhecimento estabelecido sobre a aplicação destas práticas por usuários atendidos na Atenção Primária no município de Blumenau 
Tabela 4. Análise univariada dos dados sociodemográficos e variáveis médico-assistenciais dos usuários atendidos pela Atenção Primária em Blumenau (SC).

\begin{tabular}{|c|c|c|c|c|}
\hline \multicolumn{5}{|c|}{ Uso de remédio caseiro } \\
\hline Variáveis & $\begin{array}{c}\text { Sim } \\
N(\%)\end{array}$ & $\begin{array}{c}\text { Não } \\
\text { N (\%) }\end{array}$ & $\begin{array}{c}\text { Odds Ratio } \\
\text { (IC95\%) }\end{array}$ & p \\
\hline \multicolumn{5}{|l|}{ Sexo } \\
\hline Masculino & $23(17,4)$ & $109(82,6)$ & & \\
\hline Feminino & $111(22,3)$ & $386(77,7)$ & $1,4(0,8-2,2)$ & 0,22 \\
\hline Idade (media, dp) & $50,4(16,1)$ & $43,6(18,0)$ & $1,02(1,01-1,03)$ & $<0,0001$ \\
\hline \multicolumn{5}{|l|}{ Estado civil } \\
\hline Solteiro & $19(12,9)$ & $128(87,1)$ & & \\
\hline Casado & $80(21,7)$ & $289(78,3)$ & $1,9(1,1-3,2)$ & \\
\hline Separado & $14(29,8)$ & $33(70,2)$ & $2,9(1,3-6,3)$ & \\
\hline Viúvo & $21(31,8)$ & $45(68,2)$ & $3,1(1,5-6,4)$ & 0,006 \\
\hline \multicolumn{5}{|l|}{ Cor auto-referida } \\
\hline Branca & $106(20,5)$ & $410(79,5)$ & & \\
\hline Preto/Parda/Outra & $28(24,8)$ & $85(75,2)$ & $1,3(0,8-2,1)$ & 0,320 \\
\hline \multicolumn{5}{|l|}{ Escolaridade (anos de estudo) } \\
\hline 5 anos e mais & $72(17,8)$ & $333(82,2)$ & & \\
\hline 0 a 4 anos & $62(27,7)$ & $162(72,3)$ & $1,8(1,2-2,6)$ & 0,004 \\
\hline \multicolumn{5}{|l|}{ Classe de consumo } \\
\hline $\mathrm{A} / \mathrm{B}$ & $45(21,6)$ & $163(78,4)$ & & \\
\hline $\mathrm{C}$ & $71(20,1)$ & $283(79,9)$ & $0,9(0,6-1,4)$ & \\
\hline $\mathrm{D} / \mathrm{E}$ & $18(26,9)$ & $49(73,1)$ & $1,3(0,7-2,5)$ & 0,469 \\
\hline \multicolumn{5}{|l|}{ Modalidade Assistencial } \\
\hline AG & $56(14,1)$ & $342(85,9)$ & & \\
\hline ESF & $78(33,8)$ & $153(66,2)$ & $3,1(2,1-4,6)$ & $<0,0001$ \\
\hline \multicolumn{5}{|l|}{ Tempo da doença de base } \\
\hline Até 12 meses & $48(15,2)$ & $267(84,8)$ & & \\
\hline 12 meses e mais & $86(27,4)$ & $228(72,6)$ & $2,1(1,4-3,1)$ & $<0,0001$ \\
\hline \multicolumn{5}{|l|}{ Número de medicamentos } \\
\hline 0 a 2 & $57(16,3)$ & $293(83,7)$ & & \\
\hline 3 a 4 & $41(29,9)$ & $96(70,1)$ & $2,2(1,4-3,5)$ & \\
\hline 5 e mais & $36(25,4)$ & $106(74,7)$ & $1,7(1,1-2,8)$ & 0,002 \\
\hline \multicolumn{5}{|l|}{ Medicamentos de uso contínuo } \\
\hline Não & $49(18,3)$ & $219(81,7)$ & & \\
\hline Sim & $85(23,6)$ & $276(76,5)$ & $1,4(0,9-2,0)$ & 0,111 \\
\hline \multicolumn{5}{|c|}{ Hospitalização nos últimos 12 meses } \\
\hline Não & $103(20,0)$ & $411(80,0)$ & & \\
\hline Sim & $31(27,0)$ & $84(73,0)$ & $1,5(0,9-2,3)$ & 0,101 \\
\hline
\end{tabular}

IC: intervalo de confiança; AG: Ambulatório geral; ESF: Estratégia saúde da família.

Tabela 5. Modelo de regressão logística bruta e ajustada da associação entre uso de remédios caseiros e variáveis de estudo selecionadas $(n=629)$.

\begin{tabular}{lcccc}
\hline & \multicolumn{2}{c}{ Uso de remédio caseiro } & \multicolumn{2}{c}{ Odds ratio (IC 95\%) } \\
\hline \multicolumn{1}{c}{ Variáveis } & Sim & Não & Bruto & Ajustado \\
\hline Sexo & & & & \\
$\quad$ Masculino & $23(17,4)$ & $109(82,6)$ & & \\
$\quad$ Feminino & $111(22,3)$ & $386(77,7)$ & $1,4(0,8-2,2)$ & $1,9(1,4-3,3)$ \\
Idade (media, dp) & $50,4(16,1)$ & $43,6(18,0)$ & $1,02(1,0-1,03)$ & $1,02(1,0-1,03)$ \\
Modalidade Assistencial & & & & \\
$\quad$ AG & $56(14,1)$ & $342(85,9)$ & & \\
ESF & $78(33,8)$ & $153(66,2)$ & $3,1(2,1-4,6)$ & $3,2(2,1-4,8)$ \\
\hline
\end{tabular}

Razão de Máxima Verossimilhança = 50,6 p <0,0001 GOF $\chi 2=638,8$ p = 0342. IC: intervalo de confiança; AG: ambulatório geral; ESF: estratégia saúde da família. 
(SC). O presente estudo investigou o uso de remédios caseiros e as variáveis que influenciaram o uso dos mesmos pela população atendida pelo SUS a fim de contribuir com informações importantes aos gestores municipais e à comunidade como um todo no entendimento do uso de práticas terapêuticas populares.

As características sociodemográficas da amostra se mostraram semelhantes a outros estudos realizados na atenção primária no Brasil, com predomínio de indivíduos adultos, do sexo feminino e em situação de vulnerabilidade social $^{15,16}$.

Entre as afecções tratadas mais comuns nas Unidades de Saúde, destacaram-se as "doenças do aparelho circulatório" (23,2\%), as "doenças endócrinas, nutricionais e metabólicas” (13,1\%) e os "fatores que influenciam o estado de saúde e o contato com os serviços de saúde" (8,1\%). Lima et al. ${ }^{17}$, assim como Oliveira et al. ${ }^{7}$, observaram resultado semelhante para doenças do aparelho circulatório em pessoas assistidas pelo SUS. Além disso, Piuvezam et al. ${ }^{18}$ destacam que atualmente as doenças cardiovasculares representam cerca de $40 \%$ das mortes registradas no país, particularmente entre idosos.

O número médio de medicamentos consumidos nos últimos 15 dias foi de 2,6, o qual se mostrou em consonância com outros estudos no país ${ }^{15}$. Por outro lado, a proporção de pessoas que necessitaram de internação hospitalar no último ano foi elevada $(n=130,18,7 \%)$ comparada com um estudo nacional de base populacional ${ }^{19}$, fato que pode pelo menos em parte ser explicado por se tratar de clientes de serviços de saúde, potencialmente mais doentes que a população em geral.

Alguns entrevistados relataram a substituição de medicamentos por plantas medicinais, pois "achava que podia regular com remédio caseiro, trabalhava fora e não tinha receita para comprar o medicamento, relaxamento meu mesmo", provavelmente sem o conhecimento do médico, fato relatado também por Lima et al. ${ }^{17}$. Estes casos podem representar um risco para a saúde do paciente, pois a grande maioria das plantas medicinais não tem sua eficácia comprovada, bem como as possíveis interações entre os medicamentos e as plantas medicinais devidamente relatados. Veiga Júnior et al. ${ }^{20}$ revelam que entre os danos ocasionados por algumas plantas estão a hepatotoxicidade, nefrotoxicidade, genotoxicidade, incidência aumentada de tumores, efeitos abortivos, lesão ao sistema nervoso central, entre outros. Portanto, a utilização de plantas medicinais não deve ser considerada livre de riscos.
Verificou-se neste estudo que 96\% dos entrevistados escolheram plantas medicinais como a principal terapia entre os remédios caseiros. Neste sentido, dados da OMS informam que, na busca pelo bem estar e qualidade de vida, as plantas medicinais tornaram-se uma alternativa pela sua credibilidade terapêutica e baixo custo ${ }^{2}$.

Em relação ao total de 55 plantas citadas, Oliveira et al. ${ }^{7}$ observaram o uso de 65 espécies para diferentes finalidades, porém, um número superior de usuários fazia uso das medicinais $(66,2 \%)$ em relação ao presente estudo $(21,02 \%)$.

Entre as plantas citadas, o maracujá, da espécie Passiflora officinalis, pode interagir com hipnóticos e ansiolíticos, o alho (Allium sativum) diminui os níveis plasmáticos de medicamentos usados para tratar pacientes com HIV e pode causar dermatite alérgica, náuseas e vômitos. A hortelã, da espécie Mentha spicata, é responsável por causar dermatite alérgica, o boldo, da espécie Plectranthus barbatus, pode ser nefrotóxico, enquanto o gengibre (Zingiber officinale) possui propriedades abortivas. Além disso, alho, gengibre e Ginko biloba, quando usados concomitantemente com anticoagulantes do tipo varfarina, podem alterar o tempo de coagulação ${ }^{21}$. Neste sentido, também fizeram um alerta sobre a interação medicamentosa, Oliveira e Dalla-Costa ${ }^{22}$, das plantas Hypericum perforatum, Ginkgo biloba e Panax ginseng com fármacos tradicionais.

Entre outros riscos, existe a possível toxicidade intrínseca à planta, a contaminação por agentes externos, como parasitas capazes de ocasionar doenças infecciosas, a contaminação por metais pesados e pesticidas, além da adição de fármacos com o propósito de prolongar o efeito das ervas, riscos assumidos quando estas são adquiridas em feiras livres, mercados públicos ou lojas de produtos naturais.

Neste estudo, a maioria dos entrevistados relatou que coleta as plantas no próprio quintal, nesse sentido, estudos realizados com usuários do SUS em Governador Valadares $(\mathrm{MG})^{23} \mathrm{e}$ Pelotas $(\mathrm{RS})^{7}$ encontraram resultados semelhantes, indicando, respectivamente, que 52\% e $64 \%$ das plantas utilizadas são cultivadas em quintais.

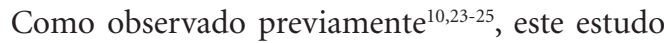
mostra de forma semelhante que a principal forma de preparo das plantas medicinais informada foi o chá. Mas, outras formas diferentes de preparo também foram mencionadas pelos usuários $(12,58 \%)$.

Entre os dez vegetais mais utilizados pelos entrevistados, seis aparecem na lista de 71 plantas que constam da Relação Nacional de Plantas 
Medicinais de Interesse ao SUS (RENISUS) ${ }^{26}$, porém apenas uma delas, a hortelã, faz parte da Relação Nacional de Medicamentos (RENAME) ${ }^{27}$. Isto demonstra que, caso Blumenau viesse a oferecer aos usuários as plantas medicinais disponíveis no RENAME, não haveria sintonia entre o material oferecido e o utilizado pela maioria da população.

Observou-se que a média de idade entre aqueles que usam alguma forma de remédio caseiro foi de 48,9 anos, e os que não usam foi de 42,1 anos, o que sugere fortemente uma associação positiva entre ambos $(p=0,0001)$. Entre os viúvos predominou o uso de remédios caseiros, fato que se mostrou relacionado, ao menos em parte, à idade encontrada entre os integrantes desse grupo, 67,89 anos $( \pm 12,92)$. A associação positiva encontrada entre o uso de remédios caseiros e a idade pode ser explicada, pelo menos em parte, devido às características da transmissão de conhecimento popular, sendo principalmente através das gerações ${ }^{17,24,28,29}$ e de forma oral, necessitando de um prolongado contato entre os indivíduos mais novos e os mais velhos, o que em comunidades urbanas é mais difícili ${ }^{29}$.

E em relação à escolaridade, indivíduos que não possuem nenhum ano de estudo utilizam mais remédios caseiros comparado com aqueles que têm 1 a 8 ou 9 e mais anos de estudo $(40,0 \%$ vs $23,2 \%$ vs $17,1 \%$ respectivamente; $\mathrm{p}=0,02)$. Arnous et al. ${ }^{30}$ e Oliveira et al. ${ }^{7}$ relataram resultado semelhante entre usuários do SUS. Outra associação significativa ocorreu entre aqueles que utilizavam mais medicamentos e também mais remédios caseiros $(p=0,0023)$. Em um estudo com idosos realizado por Leite et al. ${ }^{31}$ a maioria dos entrevistados que utilizavam remédios caseiros também usavam na mesma proporção medicamentos alopáticos. Por outro lado, em estudo recente na Alemanha, observou-se uma utilização de remédios caseiros (42\%) preferencialmente para iniciar o tratamento de doenças comuns evitando o uso de medicamentos alopáticos ${ }^{24}$.

Os resultados sugerem que pessoas do sexo feminino, com mais idade e usuárias de ESF são as principais usuárias de remédios caseiros. Esse comportamento também foi observado por Silva et al. ${ }^{32}$ e pode ser explicado, ao menos em parte, pela cobertura de ESF em Blumenau estar em áreas de maior vulnerabilidade social.

O município que desejar estimular a utilização de plantas medicinais precisa oferecer formas de capacitação para as equipes de saúde, pois apesar do Ministério da Saúde incentivar o uso de plantas medicinais e fitoterápicos, se observa que o ensino da utilização terapêutica das plantas medicinais não está comumente contemplado nos currículos das faculdades de medicina ${ }^{24} \mathrm{e}$ enfermagem, por exemplo.

Para auxiliar na reflexão, Antônio et al. ${ }^{33}$ realizaram um estudo comparando os períodos de 1990-2002 com 2003-2013 constatando que houve um pequeno aumento nas publicações relacionadas ao tema "Plantas medicinais e atenção primária à saúde". Segundo esses pesquisadores, os motivos para a pouca adesão ao uso desta terapia estariam relacionados ao baixo interesse acadêmico no tema, possivelmente devido à falta de incentivo do poder público. Outra hipótese estaria no fato de que a fitoterapia seria um conhecimento antigo e sagrado, não fazendo parte de um futuro envolvendo novas tecnologias, ou ainda, pela integração falha entre as diferentes áreas do conhecimento envolvidas. Por fim, a baixa avaliação no sistema Qualis dos periódicos que publicam sobre o tema para a área Saúde Coletiva pode indicar que o assunto não é prioridade nos periódicos científicos desta área.

Entretanto, em 2010, o Ministério da Saúde publicou portaria instituindo o programa Farmácia Viva, no âmbito dos estados e municípios, como forma de estimular o cultivo e a produção de plantas medicinais e fitoterápicos. Dessa forma, o caminho para a produção e a utilização das plantas medicinais está aberto para os municípios que demonstrarem interesse.

Observa-se um esforço, porém o caminho é longo, para colocar em prática as diretrizes determinadas para nortear o processo de implantação da PNPIC, que são, elaboração da Relação Nacional de Plantas Medicinais e da Relação Nacional de Fitoterápicos, provendo acesso à formação e educação permanente dos profissionais de saúde, acompanhamento e avaliação da inserção e implementação, fortalecimento e ampliação da participação popular e do controle social, incentivo à pesquisa e desenvolvimento, priorizando a biodiversidade do país, promoção do uso racional e garantia do monitoramento da qualidade dos fitoterápicos pelo Sistema Nacional de Vigilância Sanitária. No Brasil, muitos estados já têm utilizado plantas medicinais e fitoterápicos no $\mathrm{SUS}^{34,35}$ e, em 2010, esta prática foi introduzida em 16 estados da federação ${ }^{36}$.

\section{Conclusão}

Neste estudo foi possível verificar que a principal forma de uso de remédios caseiros pelos 
usuários do SUS em Blumenau foi com plantas medicinais. Apesar de menos utilizadas do que a medicina convencional, as plantas continuam sendo uma alternativa terapêutica para parte da população feminina, com mais idade e usuárias das ESFs. Além disso, comparando o número de plantas medicinais citadas pelos usuários neste estudo com outros similares, com mais usuários deste tipo de terapia, proporcionalmente foi maior, demonstrando conhecimento popular a ser resgatado. Ainda, estas pessoas fazem a coleta do material vegetal no próprio quintal e preparam as plantas na forma de chá. O uso provável

\section{Colaboradores}

AV Parisotto trabalhou na pesquisa, análise dos dados e redação do artigo, G Mattos participou na análise e interpretação dos dados e redação do artigo e, ALB Zeni e ET Santa Helena participaram na concepção, análise dos dados e redação final do artigo.

\section{Agradecimentos}

Este estudo fez parte do projeto "Melhoria da qualidade da Assistência Farmacêutica na atenção básica do SUS, Blumenau/SC” e contou com suporte financeiro da Organização Panamericana da Saúde. sem o conhecimento médico/especializado de qualquer droga pode ser perigoso à saúde pelos efeitos colaterais e também por sua possível interação com medicamentos, visto os usuários entrevistados fazerem uso de plantas medicinais e medicamentos alopáticos concomitantemente. Os resultados obtidos reúnem informações sobre o uso de remédios caseiros pelos usuários de serviços de Atenção Primária à Saúde em Blumenau permitindo contribuir para formulações de propostas que visem à implantação das diretrizes da PIC, incentivando o uso orientado de plantas medicinais e fitoterápicos.

\section{Referências}

1. Leal F, Schwartsmann G, Lucas HS. Medicina complementar e alternativa: uma prática comum entre pacientes com câncer. Rev Assoc Med Bras 2008; 54(6):471486.

2. Organização Mundial da Saúde (OMS). Estratégia da OMS Sobre Medicina Tradicional. 2002-2005. Genebra: OMS; 2002.

3. Jonas WB, Levin JS. Tratado de Medicina Complementar e Alternativa. São Paulo: Editora Manole Ltda; 2001.

4. Brasil. Ministério da Saúde (MS). Programa Nacional de Plantas Medicinais e Fitoterápicos. Brasília: MS; 2009.

5. Amorim LLS. Saúde e meio ambiente- Política Nacional de Práticas Integrativas e Complementares no Sistema Único de Saúde- atitude e ampliação do acesso: uma questão de direito [dissertação]. Caxias do Sul: Universidade de Caxias do Sul; 2009.

6. Minayo MCS. Contribuições da antropologia para pensar a saúde. In: Campos GWS, Bonfim JRA, Minayo MCS, Akerman M, Júnior MD, Carvalho YM, organizadores. Tratado de saúde coletiva. São Paulo, Rio de Janeiro: Hucitec, Fiocruz; 2006. p. 201-230.

7. Oliveira SGD, Moura FRR, Demarco FF, Nascente OS Del Pino FAB, Lund RG. An ethnomedicinal survey on phytotherapy with professionals and patients from Care Units in the Brazilian Unified Health System. J Ethnopharmacol 2012; 140(2):428-437.

8. Scudeller VV, Veiga JB, Jorge LHA. Etnoconhecimento de plantas de uso medicinal nas comunidades São João do Tupé e Central (Reserva de Desenvolvimento Sustentável do Tupé) In: Biotupé: Meio Físico, Diversidade Biológica e Sociocultural do Baixo Rio Negro, Amazônia Central. Santos-Silva EM, Scudeller VV, organizadores. Manaus: UEA Edições; 2009. p. 185-199. Vol. 2. 
9. Fontanella F, Speck FP, Piovezan AP, Kulkamp IC. Conhecimento, acesso e aceitação das práticas integrativas e complementares em saúde por uma comunidade usuária do Sistema Único de Saúde na cidade de Tubarão - SC. Arq Catarin Med 2007; 36(2):69-74.

10. Zeni ALB, Bosio F. O uso de plantas medicinais em uma comunidade rural de Mata Attântica- Nova Rússia, SC. Neotrop Biol Conserv 2011; 6(1):55-63.

11. Heimall J, Bielory L. Defining Complementary and Alternative Medicine in allergies and asthma: benefits and risks. Clin Rev Allergy Immunol 2004; 27(2):93-103.

12. Külkamp IC, Burin DG, Souza HMM, Silva P, Piovezan AP. Aceitação de práticas não - convencionais em saúde por estudantes de medicina da Universidade do Sul de Santa Catarina. Rev Bras Educ Méd 2007; 31(3):229235.

13. Organização Mundial da Saúde (OMS). CID-10: Classificação estatística internacional de doenças e problemas relacionados à saúde. São Paulo: Edusp; 2000.

14. Associação Brasileira de Empresas de Pesquisa (ABEP). Critério de Classificação Econômica Brasil. São Paulo: ABEP; 2009.

15. Tomasi E, Facchini LA, Thumé E, Piccini RX, Osorio A, Silveira DS, Siqueira FV, Teixeira VA, Dilélio AS, Maia MFS. Características da utilização de serviços de atenção básica à saúdenas regiões Sul e Nordeste do Brasil: diferenças por modelo de atenção. Cien Saude Colet 2011; 16(11):4395-4404.

16. Macinko J, Lima Costa MF. Access to, use of and satisfaction with health services among adults enrolled in Brazil's Family Health Strategy: evidence from the 2008 National Household Survey. Trop Med Int Health 2012; 17(1):36-42.

17. Lima SCS, Arruda GO, Renovato RD, Alvarenga MRM. Representações e usos de plantas medicinais por homens idosos. Rev Latino-Am Enferm 2012; 20(4):778786.

18. Piuvezam G Medeiros WR, Costa AV, Emerenciano FF, Santos RC, Seabra DS. Mortality from cardiovascular diseases in the elderly: Comparative analysis of two five-year periods. Arq Bras Cardiol 2015; 105(4):371380.

19. Moreira ML, Novaes HMD. Internações no sistema de serviços hospitalares, SUS e não SUS: Brasil, 2006. Rev Bras Epidemiol 2011; 14(3):411-422.

20. Veiga Júnior FV, Pinto AC, Maciel AMM. Plantas medicinais: cura segura? Quim Nova 2005; 28(3):519-528.

21. Nicoletti MA, Oliveira-Júnior MA, Bertasso CC, Caporossi PY, Tavares APL. Principais interações no uso de medicamentos fitoterápicos. Infarma 2007; 19(1/2):32-40.

22. Oliveira AE, Dalla-Costa T. Interações famacocinéticas entre as plantas medicinais Hypericum perforatum, Ginkgo biloba e Panax ginseng e fármacos tradicionais. Acta Farm Bonaer 2004; 23(4):567-578.

23. Brasileiro BG, Pizziolo VR, Matos DS, Germano AM, Jamal CM. Plantas medicinais utilizadas pela população atendida no "Programa de Saúde da Família", Governador Valadares, MG, Brasil. Rev Bras Ciênc Farm 2008; 44(4):629-630.

24. Parisius LM, Stock-Schröer B, Berger S, Hermann K, Joos S. Use of home remedies: a cross-sectional survey of patients in Germany. BMC Family Practice 2014; 15(116):1-8.
25. Meyer L, Quadros KE, Zeni ALB. Etnobotânica na comunidade de Santa Bárbara, Ascurra, Santa Catarina, Brasil. Rev Bras Bioci 2012; 10(3):258-226.

26. Brasil. Ministério da Saúde (MS). RENISUS. Relação nacional de plantas medicinais de interesse ao SUS. Espécies vegetais. [acessado 2009 maio 15]. Disponível em: http://portal.saude.gov.br/portal/arquivos/pdf/ RENISUS.pdf

27. Brasil. Ministério da Saúde (MS). Relação Nacional de Medicamentos. Brasília: MS; 2012. [acessado 2012 nov 21]. Disponível em: http://portal.saude. gov.br/portal/arquivos/pdf/anexos_rename_2012_ pt_533_11_06_2012.pdf

28. Ceolim T, Heck RM, Barbieri RL, Schwartz E, Muniz RM, Pillon CN. Medicinal plants: knowledge transmission in families of ecological farmers in southern Rio Grande do Sul. Rev Enferm Esc USP 2011; 45(1):47-54.

29. Amorozo MCM. A abordagem etnobotânica na pesquisa de plantas medicinais. In: Di Stasi LC, organizador. Plantas medicinais: arte e ciência. Um guia de estudo interdisciplinar. São Paulo: Editora da Universidade Estadual Paulista; 1996. p. 47-68.

30. Arnous AH, Santos AS, Beinner RPC. Plantas medicinais de uso caseiro - conhecimento popular e interesse por cultivo comunitário. Revista Espaço para Saúde 2005; 6(2):1-6.

31. Leite SN, Cordeiro BC, Thiesen D, Bianchini JP. Utilização de medicamentos e outras terapias antes de consulta pediátrica por usuários de unidade pública de saúde em Itajaí-SC, Brasil. Acta Farm Bonaer 2006; 25(4):608-612.

32. Silva MIG, Gondim APS, Nunes IFS, Sousa FCF. Utilização de fitoterápicos nas unidades básicas de atenção à saúde da família no município de Maracanaú (CE). Rev Bras Farmacogn 2006; 16(4):455-462.

33. Antônio GD, Tesser CD, Moretti-Pires RO. Phytotherapy in primary health care. Rev Saude Publica 2014; 48(3):541-553.

34. Wayland C. Gendering local knowledge: medicinal plant use and primary health care in the Amazon. Med Anthropol Q 2001; 15(2):171-188.

35. Wayland C. Contextualizing the politics of knowledge: physicians' attitudes toward medicinal plants. Med Anthropol Q 2003; 17(4):483-500.

36. Brasil. Ministério da Saúde (MS). Secretaria de Atenção à Saúde. Departamento de Atenção Básica. Práticas integrativas e complementares: plantas medicinais e fitoterapia na Atenção Básica. Brasília; 2012.

Artigo apresentado em 28/09/2015

Aprovado em 22/02/2016

Versão final apresentada em 24/02/2016 